\title{
Memory for temporal order in multiple sclerosis
}

\author{
WILLIAM W. BEATTY \\ Neuropsychiatric Research Institute, Fargo, North Dakota \\ and North Dakota State University, Fargo, North Dakota \\ and \\ NANCY MONSON \\ Neuropsychiatric Research Institute, Fargo, North Dakota
}

\begin{abstract}
Patients with clinically definite multiple sclerosis and age- and education-matched normal controls were given a test that allowed simultaneous evaluation of content (i.e., item) recognition memory and recency discrimination, a form of memory for temporal order. As a group, the patients performed normally on the item recognition test, but they were impaired on recency discrimination. Multiple regression analysis suggested that normal temporal order discrimination was related to both item recognition memory and performance on the Wisconsin Card Sorting Test.
\end{abstract}

Studies of patients with focal brain lesions provide evidence that recognition memory and recency discrimination are dissociable and may be subserved by distinct brain systems. Milner and colleagues (Milner, 1971; Milner, Petrides, \& Smith, 1985) have shown that patients with unilateral excisions of the frontal lobes are impaired in making judgments of relative recency but perform normally on tests of item recognition. In contrast, patients with left or right temporal lobectomies show mild and material-specific impairments on item recognition tests but perform normally on measures of recency discrimination.

More recently Sagar, Sullivan, Gabrieli, Corkin, and Growdon (1988) studied the performance of patients with Parkinson's disease (PD) on a test that simultaneously evaluated content (i.e., item) recognition and recency discrimination. They found that the PD patients' impairment on recency discrimination was disproportionately severe, compared with their modest deficit on content recognition. PD patients are known (Taylor, Saint-Cyr, \& Lang, 1986) to perform poorly on tasks such as the Wisconsin Card Sorting Test (WCST, Heaton, 1981), which are sensitive to frontal lobe lesions, especially of the dorsolateral region (Milner, 1963). Taylor et al. (1986) have proposed that the cognitive deficits of PD patients who are not demented result from damage to connections between the striatum and the frontal cortex. Therefore, Sagar et al. (1988) suggested that their PD patients' impairments on recency discrimination might have arisen from frontal lobe dysfunction, although they did not actually demonstrate that the PD patients in their study exhibited deficits on tests like the WCST and verbal fluency, classic measures

We thank Donald E. Goodkin, Marcia K. Johnson, and David P. Salmon for their helpful comments on an earlier version of this article, Donald E. Goodkin for allowing us to test his patients, and, especially, all of the patients who participated in the study. Please address correspondence and reprint requests to William W. Beatty, Neuropsychiatric Research Institute, P.O. Box 1415, Fargo, ND 58107. that are extremely sensitive to frontal dysfunction (Benton, 1968; Milner, 1963).

The pattern of neuropsychological deficits in multiple sclerosis (MS) closely resembles that observed in PD, and both diseases have been considered forms of subcortical dementia (Cummings \& Benson, 1984; Rao, 1986). When considered as a group, MS patients achieve fewer categories and make more perseverative responses and errors on the WCST than do controls (W. W. Beatty, Goodkin, P. A. Beatty, \& Monson, 1989; W. W. Beatty, Goodkin, Monson, \& P. A. Beatty, 1989). On the average, MS patients are also impaired on verbal fluency tests (W. W. Beatty, Goodkin, Monson, P. A. Beatty, \& Hertsgaard, 1988; W. W. Beatty, Goodkin, Monson, \& P. A. Beatty, 1989). On this basis, it would be expected that MS patients would be impaired on tests of recency discrimination. Since impaired recency discrimination, like poor performance on the WCST, is associated with damage to the dorsolateral frontal cortex (Milner et al., 1985), we predicted that MS patients who performed poorly on the WCST would exhibit deficits in recency discrimination, whereas patients with normal performance on the WCST would perform recency discriminations without impairment.

On the other hand, Hirst and Volpe (1982) observed impaired recency discrimination in a mixed group of amnesic patients who showed no neuropsychological evidence of frontal lobe dysfunction. This observation suggests that memory impairments might also contribute to poor recency discrimination by MS patients. In the present experiment, we tested these hypotheses, under conditions that allowed simultaneous evaluation of content recognition and recency discrimination.

\section{METHOD}

\section{Subjects}

A total of 45 patients (16 men, 29 women) with clinically definite MS (Poser et al., 1983) participated in the study. Eighteen patients carried 
the current diagnosis of chronic progressive and 12 were diagnosed as relapsing-remitting. These disease types were based on the results of the neurological examination closest in time to the neuropsychological testing and were assigned according to operational definitions that have been published elsewhere (W. W. Beatty et al., 1988; W. W. Beatty, Goodkin, P. A. Beatty, \& Monson, 1989; W. W. Beatty, Goodkin, Monson, \& P. A. Beatty, 1989). Seventeen patients had shown no significant change in neurological status during the 2 years preceding this study and were considered to have a stable disease course. Level of disability was taken as the score on the Ambulation Index (AI, Hauser et al., 1983), obtained at the time of the current neuropsychological tests. In this population, scores on the AI are highly correlated with scores on Kurtzke's (1983) Expanded Disability Status Scale $(r=.0 .96$; W. W. Beatty, Goodkin, Hertsgaard, \& Monson, 1990). At the time of testing, 10 patients were receiving ongoing treatment with muscle relaxants, 4 patients were receiving antidepressants, 7 patients were receiving the immunosuppressant azathioprine, and 7 patients were receiving anticholinergic medications for bladder control. No patient was tested while receiving corticotropin or steroid treatment for an exacerbation. Patients averaged $3.9 \pm 3.0$ on the $\mathrm{AI}$ and $11.2 \pm 9.9$ years of disease since diagnosis.

Normal controls ( 8 men, 14 women) were recruited from the community and paid for their participation. All subjects gave written informed consent after a thorough explanation of the procedures. Patients or controls with a history of alcohol or drug abuse, serious head injury, learning disability, recent or complicated heart attack, uncontrolled hypertension or metabolic disease, central nervous system diseases (other than MS for the patients), or major psychiatric illness were excluded.

\section{Materials and Procedures}

Background measures. As a measure of global cognitive functioning, all subjects were given the Screening Examination for Cognitive Impairment in Multiple Sclerosis (SECIMS; Goodkin, Beatty, \& Monson, 1990). This test is a modification of the Mini-Mental State Exam (MMSE; M. F. Folstein, S. E. Folstein, \& McHugh, 1975), which has proved insensitive for identifying cognitive impairment in MS patients (W. W. Beatty \& Goodkin, 1990). The SECIMS is identical to the MMSE, except that the number of to-be-remembered words is increased from three to seven, and the two confrontation naming items are replaced with a 15 -item version of the Boston Naming Test (Kaplan, Goodglass, \& Weintraub, 1983).

All of the patients and 18 of the controls had served in previous studies of cognitive functioning in MS (Beatty et al., 1988; W. W. Beatty, Goodkin, P. A. Beatty, \& Monson, 1989; W. W. Beatty, Goodkin, Monson, \& P. A. Beatty, 1989). During the course of these studies, their performances on the WCST had been measured. The 4 control subjects not previously studied received the WCST at the time of the present tests.

As a second measure, known to be sensitive to frontal lobe lesions (Benton, 1968), we included a letter fluency test. On this test, subjects were allowed $60 \mathrm{sec}$ to generate (orally) as many different words as possible that began with the letter F. Proper names were not allowed. The procedure was then repeated with the letters $A$ and $S$.

Recognition-recency test. This test is a simplified version of the Verbal Temporal Ordering Test (Hirst \& Volpe, 1982). A series of 163 nouns that contained three to seven letters each and had a frequency rating of at least 25 times per million (Kucera \& Francis, 1967) were printed on index cards. Subjects read each word aloud and were given approximately $2 \mathrm{sec}$ of exposure to each word. At random intervals throughout the series, index cards containing questions that probed either content recognition or recency discrimination were presented. For content recognition, the question was, "Which of these words have you seen on this test?" and the subjects had to choose between a previously presented word and a distractor (a word with the same number of letters as the correct word). For recency discrimination, the question was, "Which of these words did you see more recently?" and the subjects had to choose between two previously presented words. For both types of questions, the two alternatives were printed below the question, and the spatial location of the correct answer (left or right) varied randomly.

The test questions appeared at $1,3,6$, or 10 intervals after presentation of the stimulus word. The length of each interval was defined by the number of events that intervened between the stimulus and presentation of a test question. An event was either the presentation of a stimulus word or a test question. For recency discrimination, the intervals were defined by the number of events that intervened between presentation of each of the two stimulus words and the test question. The intervals $1-3,1-6,1-10,3-6,3-10$, and 6-10 were used. For both content recognition and recency discrimination, there were 10 questions at each interval.

The subjects were instructed to read each word aloud and to try to remember its order of occurrence. When questions were encountered, the experimenter read the question aloud and the subjects stated their answer.

\section{RESULTS}

As is shown in Table 1, the MS and control groups were well matched on age and education. The patients performed more poorly than controls did, on the SECIMS, the letter fluency test, and the WCST.

Table 2 summarizes the results of the content recognition and recency discrimination tests. The MS patients tended to perform more poorly than the controls on the content recognition test, but this difference was not statistically significant $[F(1,65)=2.50, p>.10]$. Group differences were significant, however, on the recency discrimination test $[F(1,65)=11.16, p<.001]$. On both tasks, the main effect of interval length was significant $[F \mathrm{~s}>21.15, p s<.001]$, but the groups $\times$ interval length interaction was not $\left(F_{\mathrm{s}}<1.54\right)$.

To estimate the factors that might contribute to accurate recency discrimination by MS patients, we computed product-moment correlations. Average accuracy across the six intervals was significantly related to average accuracy on the content recognition task $(r=0.53)$, total score on the SECIMS $(r=0.47)$, number of correct words on the letter fluency task $(r=0.38)$, number of categories achieved on the WCST $(r=0.37)$, and number of perseverative errors $(r=-0.37)$, but not to age or education. A stepwise multiple regression analysis indicated that accuracy on the content recognition task accounted for $26.0 \%$ of the variance in recency discrimination scores. The number of categories achieved accounted for an additional $10.3 \%$ of the variance, but none of the other factors added significantly to the variance explained.

Table 1

Demographic and Psychometric Characteristics of MS Patients and Controls: Means and Standard Deviations

\begin{tabular}{|c|c|c|c|c|c|}
\hline & \multicolumn{2}{|c|}{ Controls } & \multicolumn{2}{|c|}{ MS Patients } & \multirow{2}{*}{$\begin{array}{c}F(1,65) \\
\text { for Group }\end{array}$} \\
\hline & $M$ & $S D$ & $M$ & $S D$ & \\
\hline Age (years) & 49.0 & 16.7 & 44.9 & 11.8 & 1.36 \\
\hline Education (years) & 14.3 & 2.0 & 14.3 & 2.3 & 0.00 \\
\hline SECIMS (points) & 48.7 & 1.4 & 45.8 & 3.7 & $12.24 \dagger$ \\
\hline FAS (no. words) & 44.9 & 11.8 & 31.6 & 11.6 & $19.32 \dagger$ \\
\hline \multicolumn{6}{|l|}{ WCST } \\
\hline Categories & 5.7 & 1.1 & 4.1 & 2.2 & $9.38 *$ \\
\hline Perseverative errors & 10.3 & 7.1 & 25.1 & 21.8 & $9.54 *$ \\
\hline
\end{tabular}

Note-SECIMS $=$ Screening Examination for Cognitive Impairment in Multiple Sclerosis; FAS = letter fluency test; WCST = Wisconsin Card Sorting Test. ${ }^{*} p<.01$. $\dagger p<.001$. 
Table 2

Number of Words (out of 10 )

Correctly Recognized: Means and Standard Deviations

\begin{tabular}{crcccc}
\hline & \multicolumn{2}{c}{ Controls } & & \multicolumn{2}{c}{ MS Patients } \\
\cline { 2 - 3 } \cline { 5 - 5 } Interval & $M$ & $S D$ & & $S$ \\
\hline & & Content & & \\
1 & 10.0 & 0 & & 10.0 & 0.1 \\
3 & 9.1 & 1.1 & & 8.8 & 1.3 \\
6 & 9.0 & 0.8 & & 8.9 & 1.0 \\
10 & 9.1 & 0.8 & & 8.6 & 1.3 \\
& & Recency & & \\
$1-3$ & 10.0 & 0 & & 9.7 & 1.0 \\
$1-6$ & 10.0 & 0 & & 9.7 & 0.5 \\
$1-10$ & 10.0 & 0 & & 9.8 & 0.4 \\
$3-6$ & 8.9 & 0.8 & & 7.9 & 1.6 \\
$3-10$ & 8.2 & 1.2 & & 7.5 & 1.5 \\
$6-10$ & 6.6 & 1.2 & 6.2 & 1.5 \\
\hline
\end{tabular}

\section{DISCUSSION}

The present findings demonstrated that MS patients were impaired in memory for temporal order, but not on a concurrent test of content recognition. These results are qualitatively similar to those of Sagar et al. (1988), who observed disproportionately severe deficits in memory for temporal order among PD patients on a similar task.

Although the MS patients were not significantly impaired on the content recognition test, regression analysis indicated that accuracy of recognition memory might be an important determinant of accurate temporal order memory. A second factor, performance on the WCST, a task that is dependent on the integrity of frontal-striatal circuits (Milner, 1963; Taylor et al., 1986), was also implicated by the regression analysis.

Previous studies indicate that recency discrimination may be impaired by focal lesions of the frontal lobes, which impair performance on the WCST but leave memory relatively intact (Milner et al., 1985), or in amnesic patients without evidence of frontal lobe dysfunction (Hirst \& Volpe, 1982). On this basis, it seems reasonable to propose that the deficits in temporal order memory of MS patients are the joint product of damage to temporal lobe or diencephalic memory systems and to frontal-striatal circuits that may be generally important in sequencing (Beatty \& Monson, 1990; Sullivan, Sagar, Gabrieli, Corkin, \& Growdon, 1989).

Sagar et al. (1988) emphasized the importance of frontal lobe dysfunction in the deficits in temporal order memory for verbal stimuli that they observed in their PD patients. They did not report the correlation between content and recency discrimination, but in a later study, using nonverbal stimuli, Sullivan and Sagar (1989) reported a significant correlation $(r=0.60)$ for accuracy of content and recency discrimination by PD patients. Given the many qualitative similarities in the patterns of neuropsychological impairments in MS and PD (Cummings \& Benson, 1984; Rao, 1986), it is not unlikely that dysfunctions of primary memory systems also contribute to impaired recency discrimination by PD patients.

\section{REFERENCES}

Beatty, W. W., \& Goodkin, D. E. (1990). Screening for cognitive impairment in multiple sclerosis: An evaluation of the Mini-Mental State Exam. Archives of Neurology, 47, 297-301.

Beatty, W. W., Goodkin, D. E., Beatty, P. A., \& Monson, N. (1989). Frontal lobe dysfunction and memory impairment in patients with chronic progressive multiple sclerosis. Brain \& Cognition, 11, 73-86.

Beatty, W. W., Goodkin, D. E., Hertsgaard, D., \& Monson, N. (1990). Clinical and demographic predictors of cognitive performance in multiple sclerosis: Do diagnostic type, disease duration and disability matter? Archives of Neurology, 47, 305-308.

Beatty, W. W., Goodkin, D. E., Monson, N., \& Beatty, P. A. (1989). Cognitive disturbances in patients with relapsing remitting multiple sclerosis. Archives of Neurology, 46, 1113-1119.

Beatty, W. W., Goodkin, D. E., Monson, N., Beatty, P. A., \& HertsGAARD, D. (1988). Anterograde and retrograde amnesia in patients with chronic progressive multiple sclerosis. Archives of Neurology, 45, 611-619.

BeATTY, W. W., \& Monson, N. (1990). Cognitive and motor sequencing in Parkinson's disease. Manuscript submitted for publication.

BENTON, A. L. (1968). Differential effects of frontal lobe disease. Neuropsychologia, 6, 53-60.

Cummings, J. L., \& Benson, D. F. (1984). Subcortical dementia: Review of an emerging concept. Archives of Neurology, 41, 871-879.

Folstein, M. F., Folstein, S. E., \& McHugh, P. R. (1975). “MiniMental State": A practical method for grading the cognitive state of patients for the clinician. Journal of Psychiatry Research, 12, 189-198.

Goodkin, D. E., Beatty, W. W., \& Monson, N. (1990). Screening examination for detecting cognitive impairment in patients with multiple sclerosis. Manuscript submitted for publication.

Hauser, S. L., Dawson, D. M., Lehrich, J. R., Beal, M. F., Kevy, S. V., Propper, R. D., Mills, J. A., \& Weiner, H. L. (1983). Intensive immunosuppression in progressive multiple sclerosis. New England Journal of Medicine, 308, 173-180.

Heaton, R. K. (1981). Wisconsin Card Sorting Test manual. Odessa, FL: Psychological Assessment Resources.

HIRST, W., \&OLPE, B. T. (1982). Temporal order judgments with amnesia. Brain \& Cognition, 1, 294-306.

Kaplan, E., Goodglass, H., \& Weintraub, S. (1983). Boston Naming Test. Philadelphia: Lea \& Febiger.

KuČerA, H., \& Francis, W. N. (1967). Computational analysis of present-day American English. Providence, RI: Brown University Press.

KURTZKE, J. F. (1983). Rating neurological impairment in multiple sclerosis: An expanded disability status scale (EDSS). Neurology, 33, 1444-1452.

Milner, B. (1963). Effects of different brain lesions on card sorting. Archives of Neurology, 9, 90-100.

MILNER, B. (1972). Interhemispheric differences in the localization of psychological processes in man. British Medical Bulletin, 27, 272-277.

Milner, B., Petrides, M., \& SMith, M. L. (1985). Frontal lobes and the temporal organization of memory. Human Neurobiology, 4, 137-142.

Poser, C. M., Paty, D. W., Scheinberg, L., McDonald, W. I., Davis, F., Ebers, G. C., Johnson, K. P., Sibley, W. A., Silberberg, D. H., \& Tourtellotte, W. W. (1983). New diagnostic criteria for multiple sclerosis: Guidelines for research protocols. Annals of Neurology, 13, 227-231.

RAO, S. M. (1986). Neuropsychology of multiple sclerosis: A critical review. Journal of Clinical \& Experimental Neuropsychology, 8, 503-542.

Sagar, H. J., Sullivan, E. V., Gabrieli, J. D. E., Corkin, S., \& Growdon, J. H. (1988). Temporal ordering and short-term memory deficits in Parkinson's disease. Brain, 111, 525-539.

Sullivan, E. V., \& Sagar, H. J. (1989). Nonverbal recognition and recency discrimination deficits in Parkinson's disease and Alzheimer's disease. Brain, 112, 1503-1517.

Sullivan, E. V., Sagar, H. J., Gabrieli, J. D. E., Corkin, S., \& Growdon, J. H. (1989). Different cognitive profiles on standard behavioral tests in Parkinson's disease and Alzheimer's disease. Journal of Clinical \& Experimental Neuropsychology, 11, 799-820.

TaYlor, A. E., Saint-Cyr, J. A., \& LaNG, A. E. (1986). Frontal lobe dysfunction in Parkinson's disease: The cortical focus of neostriatal outflow. Brain, 109, 845-883.

(Manuscript received May 14, 1990.) 